at high temperatures by using low-wear, high-traction materials or coatings. Oilless traction drives will not encounter the temperature swing problems of other proposed planetary vehicle drives. Traction drives also will be less sensitive to dusty conditions if sealed properly, and will also not contaminate a planetary environment because there is no liquid lubricant used.

The oil-free traction drive is a "toothless" drive system that is capable of dry operation using low-wear, high-friction mate- rials and coatings. Multi-roller traction drive configurations offer high reduction ratios (>30 to 1 ) in a single stage, reducing motor size and providing a lightweight wheel drive system.

A traction drive with nearly pure rolling action provides much longer life than could be achieved with gear or harmonic drive systems in applications where liquid lubricants could not be used. Use of ceramic-coated titanium or polymer rollers will reduce the weight of the drives and also reduce the cost to launch.
This work was done by Richard C. Klein, Robert L. Fusaro, and Florin Dimofte of NASTEC, Inc. for Glenn Research Center. Further information is contained in a TSP (see page 1).

Inquiries concerning rights for the commercial use of this invention should be addressed to NASA Glenn Research Center, Innovative Partnerships Office, Attn: Steven Fedor, Mail Stop 4-8, 21000 Brookpark Road, Cleveland, Ohio 44135. Refer to LEW-18826-1.

\title{
* Reliable Optical Pump Architecture for Highly Coherent Lasers Used in Space Metrology Applications
}

\section{This design also can be used in scientific instrumentation where repair is difficult such as in underwater deployment.}

\section{NASA's Jet Propulsion Laboratory, Pasadena, California}

The design and initial demonstration of a laser pump module (LPM) incorporating single-mode, grating-stabilized 808-nm diode lasers and a low-loss, highport-count optical combiner are completed. The purpose of the developed LPM is to reliably pump an Nd:YAG crystal in the laser head (LH), which serves as the optical metrology source for SIMLite mission. Using the narrowlinewidth, single-mode laser diodes enables placement of the pump power near Nd adsorption peak, which enhances pumping efficiency. Grating stabilization allows for stable pump spectra as diode operating temperature and bias current change. The low-loss, high-portcount optical combiner enables efficient combining of tens of pumps. Overall, the module supports $5+$ years of continuous operation at $2 \mathrm{~W}$ of pump power with reliability approaching 100 percent.

The LPM consists of a laser diode farm (LDF) and a pump beam combiner (PBC). An array of 807- to 808-nm fiberpigtailed laser diodes makes up the LDF. A Bragg grating in each 5 - $\mu \mathrm{m}$ core single-mode (SM) fiber pigtail acts to stabilize the lasing spectra over a range of diode operating conditions. These commercially available single-mode laser diodes can deliver up to $150 \mathrm{~mW}$ of optical power. The outputs from the multiple pumps in the LDF are routed to the PBC, which is a 37 -input by 1-output allfiber device. The input ports consist of 5$\mu \mathrm{m}$ core SM fiber, while the output port consists of $105-\mu \mathrm{m}$ core, $0.15 \mathrm{NA}$ (numerical aperture) multi-mode (MM)

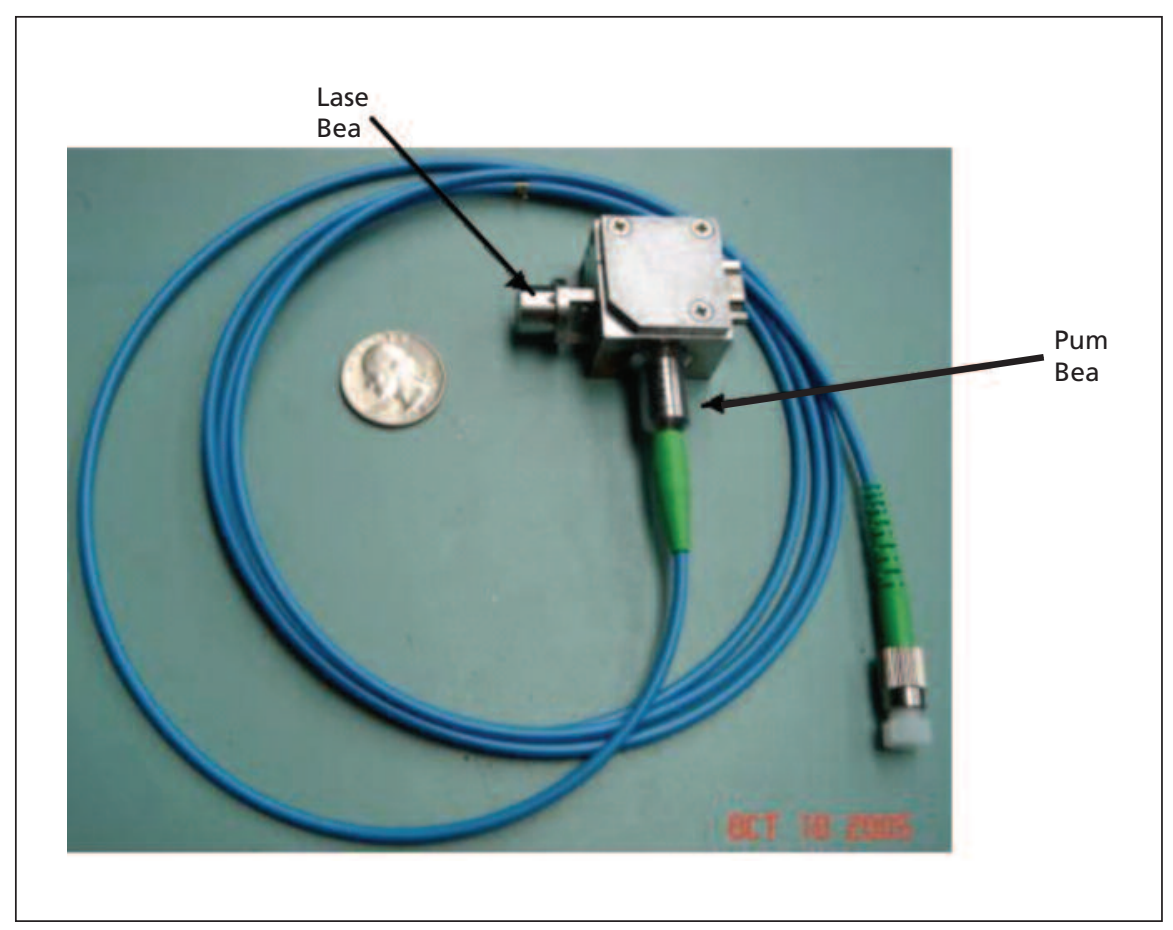

Picture of SIM-lite NPRO based LH. The optical pump is delivered to the NPRO via multimode optical fiber. The laser beam is free spaced coupled to remainder of metrology system.

fiber. The combiner is fabricated by fusing the 37 input fibers while simultaneously tapering the fused region. At the completion of this process, the MM fiber is spliced to the end of the adiabatic taper, and, for protection, the combiner is sheathed by a capillary tube. A compact and robust metal housing was designed and fabricated to protect the PBC during space deployment.
Finally, the combined pump light is delivered to the $\mathrm{LH}$ via MM optical fiber. Within the head, the pump beam optical train matches the pump beam to the lasing mode profile, which enhances pumping efficiency. The LH houses non-planar resonant oscillator (NPRO), and provides all conditioning necessary for proper NPRO operation. Two large magnets ensure unidirectional propagation through 
the ring, mitigating hole burning. A set of heaters enables slow NPRO frequency tuning, while a piezoelectric transducer enables fast tuning. LH produces $300 \mathrm{~mW}$ at $1,319 \mathrm{~nm}$ when pumped by $1.9 \mathrm{~W}$ at $808 \mathrm{~nm}$.

A software package predicting LDF reliability during the mission lifetime was developed based on the reliability theory of diode lasers. Extensive numerical simulations were carried out to select LDF architecture that meets the stringent reliability requirement ( $>99.7 \%$ probability of successful operation over 5.5 years), while balancing the competing needs to keep the number of laser diodes manageable and their operating temperature as close to room temperature as possible. Detailed sensitivity studies were also performed to make sure that the selected architecture is not vulnerable to possible deviations of key diode and mission parameters from their assumed values. The
LDF architecture that was ultimately chosen meets the reliability requirements with 37 pump laser diodes operating at $-1{ }^{\circ} \mathrm{C}$ (or $-5{ }^{\circ} \mathrm{C}$ for an extra margin of safety).

This work was done by Hernan Erlig, Yueming Qiu, Ilya Y. Poberezhskiy, Patrick L. Meras, Daniel H. Chang, and Yen-Hung Wu of Caltech for NASA's Jet Propulsion Laboratory. For more information, contact iaoffice@jpl.nasa.gov. NPO-47654 